through the center of gravity and the vertices and so is a sphere.

The statement of our main theorem can be given in more general form but our statement is chosen on account of its intuitive simplicity. The set $R$ we may take as merely closed and bounded; $S$ may be the frontier of a bounded domain, $D$, which contains $R$. Then the conclusion remains the same as we have stated it in the simpler case.

The Rice Institute

\title{
A DECOMPOSITION THEOREM FOR CLOSED SETS*
}

\section{BY G. T. WHYBURN}

Let $P$ be any local $\dagger$ topological property of a closed set such that if $K$ is any compact closed set lying in a metric space, then the set of all non- $P$-points of $K$ is either vacuous or such that its closure is of dimension $>0$. The following are examples of such properties: (i) local connectivity, (ii) regularity (MengerUrysohn sense), (iii) rationality, (iv) being of dimension $<n$, (v) belonging to no continuum of convergence, (vi) belonging to no continuum of condensation. In fact, it will be noted that in each of these cases, every non- $P$-point of a compact set $K$ lies in a non-degenerate continuum of non- $P$-points of $K$. We proceed to prove the following theorem.

Theorem. If $N$ denotes the set of all non-P-points of a compact closed set $K$ in a metric space and if $K$ is decomposed upper semi-continuously $\ddagger$ into the components of $\bar{N}$ and the points of $K-\bar{N}$, then every point of the hyperspace $H$ is a P-point of $H$.

* Presented to the Society, October 27, 1934.

$\dagger$ For the purposes of the present paper we shall understand by a local property of a set $K$ a point property $P$ such that if some neighborhood of a point $x$ in $K$ has property $P$ at $x$, then $K$ has property $P$ at $x$; and conversely, if $K$ has property $P$ at $x$, then any neighborhood of $x$ in $K$ also has property $P$ at $x$. A point $x$ of $K$ will be called a $P$-point or a non- $P$-point of $K$ according as $K$ does or does not have property $P$ at $x$.

$\ddagger$ For the notions relating to upper semi-continuous decompositions and for a proof that our particular decomposition is upper semi-continuous, the reader is referred to R. L. Moore, Foundations of Point Set Theory, American Mathematical Society, Colloquium Publications, 1932, Chapter 5. 
Proof. Let $T$ denote the continuous transformation of $K$ into $H$ associated with our decomposition, * that is, $T(K)=H$ and for each $x \epsilon H, T^{-1}(x)$ is either a component of $\bar{N}$ or a point of $K-\bar{N}$. Then clearly $T(\bar{N})$ is a set of dimension 0 . Thus if we suppose, contrary to our theorem, that $H$ has at least one non$P$-point, it follows by the condition on $P$ that the set of all non-P-points of $H$ can not be contained in $T(\bar{N})$. Hence there exists at least one point $x$ in $K-\bar{N}$ such that $T(x)=y$ is a non$P$-point of $H$. Let $U$ be a neighborhood of $x$ in $K$ such that $U \cdot \bar{N}=0$. Then on $U, T$ is a homeomorphism and $T(U)$ is a neighborhood of $y$ in $H$. Since $P$ is a local property and $x$ is a $P$-point of $K, x$ is also a $P$-point of $U$. Thus $y$ is a $P$-point of $T(U)$ as $U$ and $T(U)$ are homeomorphic; but then $y$ must be a $P$-point also of $H$, because $T(U)$ is a neighborhood of $y$ in $H$. Thus our supposition that not every point of $H$ is a $P$-point leads to a contradiction and our theorem is proved.

COROLlaRY. If $K$ is a compact metric continuum, then, choosing the properties (i)-(vi) above in turn, we find that the hyperspace of the decomposition is, respectively, (i) a locally connected continuum, (ii) a regular curve, (iii) a rational curve, (iv) a continuum of dimension $<n,(\mathrm{v})$ an hereditarily locally connected continuum, and (vi) a continuum having no continuum of condensation.

Part (i) of this corollary will be recognized at once as the well known result of $\mathrm{R}$. L. Moore to the effect that any continuum is a continuous curve with respect to its prime parts, for clearly the decomposition in this case is identically the decomposition of $K$ into its prime parts.

The UnIVersity of Virginia

* See Alexandroff, Mathematische Annalen, vol. 96 (1926), p. 555; and Kuratowski, Fundamenta Mathematicae, vol. 11 (1928), p. 169.

† See Mathematische Zeitschrift, vol. 22 (1925), p. 308. For the notion of a prime part of a continuum together with a similar result for irreducible continua, see Hahn, Sitzungsberichte der Akademie der Wissenschaften in Wien, Mathematisch-Naturwissenschaftliche Klasse, vol. 130 (1921), pp. 217-250. 